\title{
Predictive value of TNF- $a$, IFN- $\gamma$, and IL-10 for tuberculosis among recently exposed contacts in the United States and Canada
}

\author{
Mary R. Reichler ${ }^{1 *}$, Christina Hirsch², Yan Yuan ${ }^{1}$, Awal Khan', Susan E. Dorman ${ }^{3}$, Neil Schluger ${ }^{4}$,
}

Timothy R. Sterling ${ }^{5}$ and the Tuberculosis Epidemiologic Studies Consortium Task Order 2 Team

\begin{abstract}
Background: We examined cytokine immune response profiles among contacts to tuberculosis patients to identify immunologic and epidemiologic correlates of tuberculosis.

Methods: We prospectively enrolled 1272 contacts of culture-confirmed pulmonary tuberculosis patients at 9 United States and Canadian sites. Epidemiologic characteristics were recorded. Blood was collected and stimulated with Mycobacterium tuberculosis culture filtrate protein, and tumor necrosis factor (TNF-a), interferon gamma (IFN- $\gamma$ ), and interleukin 10 (IL-10) concentrations were determined using immunoassays.

Results: Of 1272 contacts, 41 (3.2\%) were diagnosed with tuberculosis before or $\leq 30$ days after blood collection (co-prevalent tuberculosis) and 19 (1.5\%) during subsequent four-year follow-up (incident tuberculosis). Compared with contacts without tuberculosis, those with co-prevalent tuberculosis had higher median baseline TNF-a and IFN- $\gamma$ concentrations (in pg/mL, TNF-a 129 versus 71, $P<.01$; IFN- $\gamma 231$ versus 27, $P<.001$ ), and those who subsequently developed incident tuberculosis had higher median baseline TNF-a concentrations (in pg/mL, 257 vs. $71, P<.05)$. In multivariate analysis, contact age $<15$ years, US/Canadian birth, and IFN or TNF concentrations $>$ the median were associated with co-prevalent tuberculosis $(P<.01$ for each); female sex $(P=.03)$ and smoking $(P<.01)$ were associated with incident tuberculosis. In algorithms combining young age, positive skin test results, and elevated CFPS TNF- $a$, IFN- $\gamma$, and IL-10 responses, the positive predictive values for co-prevalent and incident tuberculosis were 40 and $25 \%$, respectively.
\end{abstract}

Conclusions: Cytokine concentrations and epidemiologic factors at the time of contact investigation may predict co-prevalent and incident tuberculosis.

Keywords: Tuberculosis, Contacts, Cytokines, Immune correlates, Epidemiology, Tumor necrosis factor, Interferon gamma, Interleukin 10

\footnotetext{
* Correspondence: reichlermrr31@gmail.com

${ }^{1}$ National Center for HIV/AIDS, Viral Hepatitis, STD, and TB Prevention, Division of Tuberculosis Elimination, Centers for Disease Control and Prevention, Mailstop E-10, 1600 Clifton Road NE, 30329-4027 Atlanta, GA, USA Full list of author information is available at the end of the article
}

(c) The Author(s). 2020 Open Access This article is licensed under a Creative Commons Attribution 4.0 International License, which permits use, sharing, adaptation, distribution and reproduction in any medium or format, as long as you give appropriate credit to the original author(s) and the source, provide a link to the Creative Commons licence, and indicate if changes were made. The images or other third party material in this article are included in the article's Creative Commons licence, unless indicated otherwise in a credit line to the material. If material is not included in the article's Creative Commons licence and your intended use is not permitted by statutory regulation or exceeds the permitted use, you will need to obtain permission directly from the copyright holder. To view a copy of this licence, visit http://creativecommons.org/licenses/by/4.0/ The Creative Commons Public Domain Dedication waiver (http://creativecommons.org/publicdomain/zero/1.0/) applies to the data made available in this article, unless otherwise stated in a credit line to the data. 


\section{Background}

Tuberculosis (TB) is the leading infectious cause of death worldwide, with $\geq 10$ million new cases annually and 1.5 million deaths [1]. A better understanding of the immunologic and epidemiologic profiles associated with Mycobacterium tuberculosis infection and disease after TB exposure can help focus TB control efforts. Identification of surrogate markers of protective immunity against $M$. tuberculosis infection and TB disease can also aid development of new TB vaccines.

Immune responses among persons with $\mathrm{TB}$ disease have been the subject of multiple studies. Interferongamma (IFN- $\gamma$ ) and tumor necrosis factor alpha (TNF$\alpha)$ are essential for protection against mycobacterial infections [2]. TNF- $\alpha$ response to $M$. tuberculosis antigens may be elevated and IFN- $\gamma$ response depressed at the time of TB diagnosis, with a later decline in TNF- $\alpha$ and elevation of IFN- $\gamma$ associated with successful resolution of disease [3-5]. Further, an increase in TNF- $\alpha$ has been observed among persons with previously treated TB disease at the time of relapse [5]. Interleukin 10 (IL-10) may impair host resistance to $M$. tuberculosis infection [6]. IL-10 is known to be elevated early in the course of TB disease, and is believed to function as an inhibitor to regulate and help prevent the potentially destructive effects of an over vigorous immune response [7].

IFN- $\gamma$ response is well established as an immune correlate of $M$. tuberculosis infection. A study from Uganda reported that elevation in IFN- $\gamma$ responses are predictive of subsequent tuberculin skin test (TST) conversion in Bacillus Calmette-Guérin (BCG) vaccinated but not among unvaccinated household contacts [8]. Elevated IFN- $\gamma$ response has been closely correlated with a positive TST and TB exposure intensity, and thus by inference, with latent $M$. tuberculosis infection [9-13].

Evidence from recent studies reveals that IFN- $\gamma$ responses can also be predictive of subsequent TB disease. In an intriguing study from Colombia, investigators observed a trend toward increased risk for TB disease among contacts with upper quartile elevations of IFN- $\gamma$ [14]. In a study of contacts in Germany, a higher predictive value for TB was observed among contacts with positive IFN- $\gamma$ release assays (IGRA) compared with TST [15]. In a cohort study in South Africa, IGRA conversion from negative to positive among adolescents was correlated with a positive predictive value (PPV) for subsequent $\mathrm{TB}$ disease of $2.8 \%$ [16, 17]. In a 2012 metaanalysis, positive IGRAs had a PPV for TB of $2.7 \%$ [18]. Longitudinal studies of exposed contacts have not had sufficient power to determine whether measurable differences in IFN- $\gamma$ responses can predict which persons with latent TB infection will ultimately develop TB disease. Additionally, direct comparisons of IFN- $\gamma$ profiles among persons with TB disease and those having latent
TB infection are rare, and information on cytokine responses other than IFN- $\gamma$ is limited. Recently, a transcriptomic signature of progression from latent to active TB with a sensitivity of $39-54 \%$ was reported [19].

We conducted a prospective study of contact investigations at health departments in the United States and Canada. We previously described rates, timing, and risk factors for TB disease among contacts $[20,21]$. Here, we examine the immune response profiles of 3 cytokines believed to influence immunity to TB-TNF- $\alpha$, IFN- $\gamma$, and IL-10-in the same contact cohort.

\section{Methods}

We prospectively enrolled culture-confirmed adult TB patients and their close contacts at 9 US and Canadian sites participating in the Tuberculosis Epidemiologic Studies Consortium [22] (see published Methods [20]). Contacts were identified, enrolled, interviewed, offered human immunodeficiency virus (HIV) testing, and screened for LTBI and TB from 2002 to 2006, then followed with $\mathrm{TB}$ registry matches performed annually for 4 years after last site enrollment at 8 sites and annually for 2 years at one site (final follow-up February 2011) [20]. Since enrollment occurred over a 4-year period, contacts enrolled earlier in the study had a longer tuberculosis registry match observation period, with $100 \%$ observed for at least 4 years and $94 \%$ for at least 5 years after exposure [20]. HIV prevalence in the parent study contact cohort was 2\% [23]. While a standard protocol was used for conducting contact investigations, the staff at the study sites did not use a standard protocol for patient management, which included efforts to prevent secondary cases by investigation and treatment of contacts with LTBI [20]. Exposure hours were calculated by careful interview of both the index patient and each contact to determine hours per day, week, and month of shared indoor living space. These were then added over the course of the entire infectious period to determine the total number of exposure hours. The date of $\mathrm{TB}$ diagnosis was defined as the start date for $\mathrm{TB}$ treatment.

Contacts enrolled in the contact investigation study were offered enrollment in the immunology portion of the study at the time of contact investigation using written informed consent; contacts with incomplete TST screening, a history of prior positive TST or TB, or a positive HIV test result were ineligible and excluded from enrollment. Of 3221 eligible contacts, 1272 (39\%) consented and were enrolled. Blood for cytokine studies was collected at the time of contact investigation. Contacts with TB diagnosed before or $\leq 30$ days after blood draw were considered co-prevalent cases, and those diagnosed > 30 days after the blood draw incident cases. 
All health departments defined negative TSTs as $<5$ $\mathrm{mm}$ and positive TSTs as $>5 \mathrm{~mm}$ induration. TST conversion was defined as a first TST $<5 \mathrm{~mm}$ and a subsequent TST $\geq 5 \mathrm{~mm}$.

Whole blood was collected in heparinized tubes at the study sites and shipped to Case Western Reserve University (Cleveland, Ohio) overnight at room temperature, then diluted 10-fold with RPMI-1640 medium and dispensed into 24-well tissue culture plates. Wells remained un-stimulated or they received M. tuberculosis (H37Rv) culture filtrate protein (CFP) $(5 \mu \mathrm{g} / \mathrm{mL})$ or phytohemagglutinin (PHA) $(10 \mu \mathrm{g} / \mathrm{mL})$. Cell-free supernatants were collected after 24-h (TNF- $\alpha$ ) and 5-day (IFN- $\gamma$ and IL-10) incubation at $37^{\circ} \mathrm{C}$ with $5 \% \mathrm{CO}_{2}$ and stored frozen at $-70^{\circ} \mathrm{C}$ until use. TNF- $\alpha$, IFN- $\gamma$, and IL-10 levels in thawed supernatants were assessed by using enzyme linked immunosorbent assay kits from Endogen, R\&D Systems and Biosource, respectively. The lower limit of detection for the assays was $<2 \mathrm{pg} / \mathrm{mL},<5 \mathrm{pg} / \mathrm{mL}$ and $<$ $0.2 \mathrm{pg} / \mathrm{mL}$, respectively.

Epidemiologic characteristics and CFP-stimulated (CFPS) TNF- $\alpha$, IFN- $\gamma$, and Il-10 concentrations were evaluated for enrolled contacts. Univariate analyses were performed using $\chi^{2}$ or Fisher's exact test. Multivariate logistic regression was performed by using backward elimination. SAS 9.1 was used for all analyses. $P$-values $<.05$ were considered statistically significant.

Contacts with known HIV infection were excluded because of concerns that HIV might affect their immune responses and the risk for developing TB disease.

The protocol was approved by the institutional review boards at CDC and all participating project sites.

\section{Results}

\section{Study population}

We enrolled 1272 contacts of $718 \mathrm{~TB}$ patients. Characteristics of the study participants are presented in Table 1. A total of 60 (4.7\%) had TB disease, including 41 with co-prevalent $\mathrm{TB}$ and 19 with incident TB; 93 (7.3\%) TST conversion without TB; 502 (39.5\%) initial positive TST without TB; and 617 (49.5\%) negative TST without TB. Of the 19 contacts with incident TB, the median time from blood draw to diagnosis of $\mathrm{TB}$ was 10 months (range, 2-35 months).

Compared with non-enrolled contacts, enrolled contacts had a similar distribution of screening outcomes and similar demographic characteristics, with the exception of a lower proportion aged $<15$ years (data not shown).

\section{Cytokine responses}

Median cytokine concentrations are presented in Table 2.
CFPS TNF- $\alpha$ and IFN- $\gamma$ responses were similar for contacts with co-prevalent $\mathrm{TB}$ and for those who were TST-positive without $\mathrm{TB}$, and substantially higher in each of these groups compared with contacts who were TST-negative without TB. CFPS TNF$\alpha$ responses were also substantially higher among contacts with incident TB compared with TSTpositive contacts without TB; no substantial difference occurred in CFP IFN- $\gamma$ responses between the 2 groups. CFPS IL-10 responses were low across all outcome groups.

Tables 3 and 4 present median CFPS TNF- $\alpha$ and IFN$\gamma$ responses for contacts without TB, contacts with coprevalent $\mathrm{TB}$, and contacts with incident $\mathrm{TB}$.

Compared with contacts without $\mathrm{TB}$, contacts with co-prevalent TB had higher median CFPS TNF- $\alpha$ and IFN- $\gamma$ concentrations. Compared with contacts without $\mathrm{TB}$, contacts with incident TB had higher median CFPS TNF- $\alpha$ concentrations but no substantial difference in median CFPS IFN- $\gamma$ concentrations. CFP IL-10 responses were low across all outcome groups.

To examine CFPS cytokine responses by epidemiologic and clinical factors potentially related to outcome, the cytokine data were stratified by these factors (Tables 3 and 4).

CFPS TNF- $\alpha$ and IFN- $\gamma$ responses were substantially higher for contacts with co-prevalent TB compared with those without TB when stratified by most of the epidemiologic and clinical factors.

Among study participants without TB, CFPS TNF- $\alpha$ and IFN- $\gamma$ responses were lower for persons with diabetes mellitus, current steroid use, heavy alcohol consumption, injection drug use, or street drug use compared with persons without these conditions; some but not all differences were statistically significant. Further, among persons with one of these conditions, CFPS TNF- $\alpha$ and IFN- $\gamma$ responses were similar for contacts with and without TB.

Among study participants without TB, CFPS TNF- $\alpha$ responses were higher and CFPS IFN- $\gamma$ responses lower among persons of black versus other race/ethnicities.

CFPS IL-10 responses were low for contacts with and without TB (data not shown).

All cytokine response findings were similar when analyses were repeated excluding 385 contacts with partial or complete treatment for LTBI (data not shown).

\section{Multivariate analysis}

In multivariate analysis, age $<15$ years, US/Canadian birth, more exposure hours, and TNF- $\alpha$ or IFN- $\gamma$ CFPS concentrations greater than the median were associated with co-prevalent TB. Female sex and smoking were associated with incident TB (Table 5). 
Table 1 Epidemiologic and Clinical Characteristics of the Study Population

\begin{tabular}{|c|c|c|c|c|c|}
\hline \multirow[t]{3}{*}{ Characteristic $^{b}$} & \multirow{3}{*}{$\begin{array}{l}\text { All Contacts } \\
N=1272 \\
\underline{\mathrm{n}(\%)}\end{array}$} & \multicolumn{4}{|c|}{ Contact Outcome Group } \\
\hline & & $\begin{array}{l}\text { TB disease } \\
N=60\end{array}$ & $\begin{array}{l}\text { Converter } \\
N=93\end{array}$ & $\begin{array}{l}\text { TST }^{a} \\
N=502\end{array}$ & $\begin{array}{l}\text { TST- }^{\mathrm{b}} \\
N=617\end{array}$ \\
\hline & & $\underline{\mathrm{n}(\%)}$ & $\underline{\mathrm{n}(\%)}$ & $\underline{\mathrm{n}(\%)}$ & $\underline{\mathrm{n}(\%)}=$ \\
\hline \multicolumn{6}{|l|}{ Age (yrs) } \\
\hline $0-14$ & $136(11)$ & $8(13)$ & $8(9)$ & $25(5)$ & $95(15)$ \\
\hline $15-24$ & $300(24)$ & $16(27)$ & $20(22)$ & $131(26)$ & $133(22)$ \\
\hline $25-44$ & $460(36)$ & $18(30)$ & $35(38)$ & $198(39)$ & $209(34)$ \\
\hline $45-64$ & $304(24)$ & $18(20)$ & $24(26)$ & $116(23)$ & $146(24)$ \\
\hline$>=65$ & $72(6)$ & $0(0)$ & $6(6)$ & $32(6)$ & $34(5)$ \\
\hline \multicolumn{6}{|l|}{ Sex } \\
\hline Male & $678(53)$ & $42(70)$ & $47(50)$ & $310(62)$ & $279(45)$ \\
\hline Female & $594(47)$ & $18(30)$ & $46(50)$ & $192(38)$ & $338(55)$ \\
\hline \multicolumn{6}{|l|}{ Race } \\
\hline White & $155(12)$ & $6(10)$ & $10(11)$ & $37(7)$ & $102(17)$ \\
\hline Black & $630(50)$ & $20(33)$ & $47(51)$ & $211(42)$ & $352(57)$ \\
\hline Other & $487(38)$ & $34(57)$ & $36(39)$ & $254(51)$ & $163(26)$ \\
\hline \multicolumn{6}{|l|}{ Birthplace } \\
\hline US/Canada & $842(66)$ & $30(50)$ & $60(64)$ & $220(44)$ & $532(86)$ \\
\hline Other & $430(34)$ & $30(50)$ & $33(36)$ & $282(56)$ & $85(14)$ \\
\hline \multicolumn{6}{|l|}{$H I V^{a}$} \\
\hline Negative & $671(53)$ & $50(83)$ & $55(59)$ & $256(51)$ & $310(50)$ \\
\hline Unknown & $601(47)$ & $10(17)$ & $38(41)$ & $246(49)$ & $307(50)$ \\
\hline \multicolumn{6}{|c|}{ Location of exposure } \\
\hline Household & $812(64)$ & $26(43)$ & $73(78)$ & $341(68)$ & $372(60)$ \\
\hline School & $44(3)$ & $0(0)$ & $2(2)$ & $22(4)$ & $20(3)$ \\
\hline Social & $286(22)$ & $12(20)$ & $12(13)$ & $86(17)$ & $176(29)$ \\
\hline Workplace & $130(10)$ & $22(37)$ & $6(6)$ & $53(11)$ & $49(8)$ \\
\hline \multicolumn{6}{|l|}{ Hours of exposure } \\
\hline Median (IQR) ${ }^{a}$ & $368(171-820)$ & $634(315-768)$ & 400 (177-964) & 416 (195-920) & $300(167-710)$ \\
\hline
\end{tabular}

aefinitions: IQR interquartile range, with 25 and $75 \%$ values displayed, TST tuberculin skin test, TB tuberculosis, IQR interquartile range

${ }^{\mathrm{b}}$ All risk variables were self-reported

\section{Algorithms}

Tables 6 and 7 shows the sensitivity, specificity, positive predictive value (PPV), and negative predictive value (NPV) for co-prevalent TB and incident TB of CFPS cytokine responses.

CFPS TNF- $\alpha$, IFN- $\gamma$, and IL-10 responses greater than the median each had sensitivities for co-prevalent TB of 59-79\%, with specificities of $49-51 \%$. When all 3 CFPS cytokine responses were greater than the median, the specificity increased to $80 \%$ but the sensitivity decreased to $37 \%$. The PPV and NPV for all three CFPS cytokine responses greater than the median were 6 and 97\%, respectively.
Among TST-positive contacts with results available for all 3 cytokines, CFPS responses greater than the median for each of the three cytokines individually had a PPV for co-prevalent TB of 7-9\%, with a PPV of 9\% for all three CFPS responses $>$ median. Among TSTpositive children aged $<15$ years of age, the PPV for coprevalent TB of CFPS responses > median for TNF- $\alpha$, IFN- $\gamma$, and IL-10 individually were 16,17 , and $17 \%$, respectively, and the PPV for all three cytokine CFPS responses greater than the median was $40 \%$.

The sensitivity and specificity of CFPS TNF- $\alpha$, IFN- $\gamma$, and IL-10 responses greater than the median for incident TB were similar to those for co-prevalent TB. The 
Table 2 Median (IQR) Cytokine Responses in pg/ml Among Contacts By Outcome Group

\begin{tabular}{|c|c|c|c|c|c|}
\hline \multirow[t]{2}{*}{ Characteristic } & \multicolumn{5}{|c|}{ Contact Outcome Group } \\
\hline & $\begin{array}{l}\text { Co-prevalent TB } \\
\underline{(n=41)}\end{array}$ & $\begin{array}{l}\text { Incident TB } \\
(\underline{n}=19)\end{array}$ & $\begin{array}{l}\text { Converter } \\
(n=93) \\
\end{array}$ & $\begin{array}{l}\text { TST }+ \\
\underline{(n=502)} \\
\end{array}$ & $\begin{array}{l}\text { TST- } \\
\underline{(n=617)_{E}}\end{array}$ \\
\hline \multicolumn{6}{|l|}{ TNF-a } \\
\hline \multirow[t]{2}{*}{ Unstimulated } & 0 & 0 & 0 & 0 & 0 \\
\hline & $(0-4)$ & $(0-0)$ & $(0-4)$ & $(0-2)$ & $(0-3)$ \\
\hline \multirow[t]{2}{*}{ PHA } & 8328 & - & 4204 & 4660 & 4737 \\
\hline & $(4664-9012)$ & & $(3612-4662)$ & (2854-6679) & $(2921-8131)$ \\
\hline \multirow[t]{2}{*}{ M. tb culture filtrate protein } & $129^{a}$ & $257^{a}$ & 48 & $103^{a}$ & 50 \\
\hline & $(28-1326)$ & $(67-2011)$ & $(0-246)$ & $(20-383)$ & $(0-300)$ \\
\hline \multicolumn{6}{|l|}{ IFN- $\gamma$} \\
\hline \multirow[t]{2}{*}{ Unstimulated } & 0 & 0 & & 0 & 0 \\
\hline & $(0-4)$ & $(0-0)$ & $(0-1)$ & $(0-4)$ & $(0-1)$ \\
\hline \multirow[t]{2}{*}{ PHA } & $9988^{\mathrm{a}}$ & $1238^{\mathrm{a}}$ & 21,165 & 13,842 & 18,986 \\
\hline & $(151-15,690)$ & $(0-14,256)$ & $(4430-58,356)$ & $(674-55,046)$ & $(981-62,161)$ \\
\hline \multirow[t]{2}{*}{ M. tb culture filtrate protein } & $231^{\mathrm{a}}$ & 88 & 32 & $130^{\mathrm{a}}$ & 0 \\
\hline & $(61-549)$ & $(0-445)$ & $(0-302)$ & $(0-771)$ & $(0-130)$ \\
\hline \multicolumn{6}{|l|}{ IL-10 } \\
\hline \multirow[t]{2}{*}{ Unstimulated } & 0 & 0 & 0 & 0 & 0 \\
\hline & $(0-2)$ & $(0-0)$ & $(0-1)$ & $(0-1)$ & $(0-1)$ \\
\hline \multirow[t]{2}{*}{ M. tb culture filtrate protein } & 8 & 14 & 6 & 7 & 6 \\
\hline & (0-139) & $(3-88)$ & $(1-19)$ & $(1-30)$ & $(0-28)$ \\
\hline
\end{tabular}

${ }^{\mathrm{a}} P<.05$ vs. TST- group; $\varnothing P<.05$ comparing Incident TB vs. TST+ group; $\Delta \mathrm{P}<.05$ comparing Co-prevalent TB vs. TST+ group

Definitions: PHA Phytohemagglutinin. TB Tuberculosis, TST Tuberculin skin test, TNF Tumor necrosis factor, IFN Interferon gamma; IL-10 = Interleukin 10; $M$. tb= Mycobacterium tuberculosis; Co-prevalent TB defined as TB diagnosis before or $<30$ days after blood draw; Incident TB defined as TB diagnosis $>30$ days after blood draw

Clarifications: PHA stimulation not performed for IL-10; the number of contacts included varies slightly from the numbers presented in the heading because 14 contacts were missing TNF culture filtrate protein results, 38 contacts were missing IFN culture filtrate protein results, and 23 contacts were missing IL10 culture filtrate protein results

PPVs and NPVs for incident TB for all 3 CFPS cytokine responses greater than the median were 4 and $99 \%$, respectively. Among TST-positive children aged $<15$ years of age, the PPV for incident TB of all three cytokine CFPS responses $>$ median was $25 \%$.

\section{Discussion}

In our study, CFPS cytokine responses were evaluated for contacts after exposure to an infectious TB patient. A total of 60 exposed contacts were diagnosed with TB disease, representing $4.7 \%$ of all enrolled contacts; for 19 of these, specimens were collected from two months to three years before TB diagnosis. The majority of previous reports have been limited to assessment of CFPS cytokine responses after rather than before TB diagnosis $[3-5,7,9,24-26]$. Further, most previous studies have included limited numbers of TB patients and a convenience sample of healthy control subjects. The detailed epidemiologic data collected prospectively on our study population provide a unique opportunity to evaluate immune correlates of protection, infection, and disease.
CFPS TNF- $\alpha$ and IFN- $\gamma$ responses were significantly higher for contacts with co-prevalent TB than for contacts without TB. In multivariate analyses that examined CFPS cytokine responses and epidemiologic factors related to exposure and TB disease risk, elevated CFP IFN- $\gamma$ or TNF- $\alpha$ responses were independent predictors of co-prevalent TB. These findings indicate that CFPS TNF- $\alpha$ and IFN- $\gamma$ responses might be useful tools for predicting active $\mathrm{TB}$ and prompt consideration of their use during contact investigation.

Our study also identified algorithms based on immunologic and epidemiologic factors with higher PPVs for subsequent incident TB disease than reported for IFN- $\gamma$ release assays $(2.7 \%)$ or TSTs (1.5\%) [18]. Transcriptomic signatures have also shown promising results, but have not been tested in combination with clinical and epidemiologic factors; further, reports to date have included co-prevalent TB as well as incident TB cases $[19,27]$. In an algorithm combining young age, positive TST results, and elevated CFPS TNF- $\alpha$, IFN- $\gamma$, and IL10 responses, the estimate of PPV for incident TB disease in our study was $25 \%$, which was high compared 
Table 3 Median (IQR) $)^{b}$ TNF-a ${ }^{b}$ Responses to M. tuberculosis Culture Filtrate Protein in pg/ml By Clinical and Epidemiologic Characteristics and Outcome Group

\begin{tabular}{|c|c|c|c|c|c|}
\hline \multirow[t]{2}{*}{ Characteristic $^{c}$} & \multicolumn{3}{|c|}{ Contact Outcome Group } & \multirow{2}{*}{$\begin{array}{l}\text { P-value } \\
\text { Co-prevalent TB } \\
\text { vs. Not TB } \\
\end{array}$} & \multirow{2}{*}{$\begin{array}{l}\text { P-value } \\
\text { Incident TB } \\
\text { vs. Not TB } \\
\end{array}$} \\
\hline & $\begin{array}{l}\text { Co-prevalent } \mathrm{TB}^{\mathrm{d}} \\
(\underline{n}=41)\end{array}$ & $\begin{array}{l}\text { Incident TBd } \\
(\underline{n=19)}\end{array}$ & $\begin{array}{l}\text { Not TBd } \\
(n=1212)\end{array}$ & & \\
\hline All & $129(28-1326)$ & 257 (67-2011) & $71(1-336)$ & 0.007 & 0.037 \\
\hline \multicolumn{6}{|l|}{ Age (yrs) } \\
\hline $0-14$ & $72(19-3964)$ & 237 (237-237) & $120(10-1026)$ & 0.71 & 0.31 \\
\hline $15-24$ & $83(28-1491)$ & 549 (120-549) & $77(12-744)$ & 0.51 & 0.10 \\
\hline $25-44$ & $217(107-815)$ & $224(16-548)$ & $79(11-322)$ & 0.08 & 0.41 \\
\hline $45-64$ & $96(42-129)$ & $1039(0-2471)$ & $39(0-189)^{e}$ & 0.08 & 0.41 \\
\hline$>=65$ & NA & NA & $52(0-247)^{f}$ & $N A^{a}$ & $N A^{a}$ \\
\hline \multicolumn{6}{|l|}{ Sex } \\
\hline Male & $129(28-1325)$ & $247(44-1280)$ & $77(13-335)$ & 0.02 & 0.13 \\
\hline Female & $83(21-217)$ & 548 (67-2021) & $61(0-336)^{f}$ & 0.07 & 0.08 \\
\hline \multicolumn{6}{|l|}{ Race/Ethnicity } \\
\hline Black & $2042(19-5053)$ & $1124(0-2471)$ & $80(4-606)$ & 0.52 & 0.52 \\
\hline Non-black & $96(28-305)$ & $257(120-549)$ & $60(0-242)^{e}$ & 0.006 & 0.003 \\
\hline \multicolumn{6}{|l|}{ Birthplace } \\
\hline US/Canada & $96(23-3300)$ & $257(16-2010)$ & $57(0-358)$ & 0.09 & .16 \\
\hline Other & $132(56-815)^{f}$ & $334(73-549)$ & $94(21-308)^{e}$ & 0.21 & 0.41 \\
\hline BCG- & $90(10-1325)$ & $393(8-2446)$ & $62(1-358)$ & 0.41 & 0.25 \\
\hline BCG+ & $132(129-305)^{\mathrm{e}}$ & $257(120-549)$ & $79(13-244)^{f}$ & 0.01 & 0.06 \\
\hline \multicolumn{6}{|l|}{ HIV status ${ }^{\mathrm{b}}$} \\
\hline HIV negative & $129(28-784)$ & $257(67-549)$ & $60(0-295)$ & 0.007 & 0.03 \\
\hline HIV unk & $107(37-3632)$ & $4492(237-8748)$ & $80(4-408)^{e}$ & 1.00 & 0.16 \\
\hline \multicolumn{6}{|l|}{ Diabetes mellitus } \\
\hline Yes & $N A^{a}$ & $67(0-257)^{f}$ & $39(0-192)$ & $N A^{a}$ & 0.56 \\
\hline No & $129(50-1326)$ & $549(97-2016)^{f}$ & $73(4-358)^{f}$ & 0.004 & 0.04 \\
\hline \multicolumn{6}{|l|}{ Steroids $^{\mathrm{b}}$} \\
\hline Yes & $10(0-21)$ & NA & $89(6-179)$ & 0.15 & NA \\
\hline No & $129(80-1326)^{f}$ & 257 (67-2011) & $71(1-358)$ & 0.001 & 0.037 \\
\hline \multicolumn{6}{|c|}{ Smoking past 6 months } \\
\hline Yes & 305 (96-1491) & $403(120-549)$ & $57(0-305)$ & 0.01 & 0.03 \\
\hline No & $129(76-1055)$ & $73(67-2011)$ & $77(5-352)^{f}$ & 0.01 & 0.65 \\
\hline \multicolumn{6}{|l|}{ Heavy alcohol ${ }^{b}$} \\
\hline Yes & $0(0-83)$ & $334(68-4648)$ & 77 (20-595) & 0.57 & 0.31 \\
\hline No & $129(80-1325)^{f}$ & 257 (67-2011) & $68(1-305)$ & 0.002 & 0.07 \\
\hline \multicolumn{6}{|l|}{ IV drugs ${ }^{\mathrm{b}}$} \\
\hline Yes & $135(135-135)$ & $191(191-191)$ & $37(0-166)$ & 0.32 & 0.32 \\
\hline No & $129(28-1326)$ & 403 (67-2011) & $71(1-347)^{f}$ & 0.006 & 0.06 \\
\hline \multicolumn{6}{|l|}{ Street drugs ${ }^{b}$} \\
\hline Yes & $135(83-7330)$ & $0(0-191)$ & $50(4-234)$ & 0.08 & 0.56 \\
\hline No & $129(28-1325)$ & $549(97-2016)^{f}$ & $76(1-361)^{\mathrm{e}}$ & 0.01 & 0.04 \\
\hline
\end{tabular}

${ }^{\mathrm{a}} \mathrm{NA}$ (not applicable) in place of a cytokine response designates that there were no specimens for testing; NA in place of a p-value designates that a comparison could not be made

${ }^{\mathrm{b}}$ Co-prevalent TB defined as TB diagnosis before or $<30$ days after blood draw; Incident TB defined as TB diagnosis $>30$ days after blood draw; TNF- $\mathrm{a}=$ Tumor necrosis factor alpha; Steroids = current steroid use; IV drug = current intravenous drug use; HIV=Human Immunodeficiency Virus; Heavy alcohol = current consumption of $>12$ beers or $>1$ bottle of wine or $>1$ pint of hard liquor per week; Street drugs = current use of drugs other than intravenous acquired without a prescription; $\mathrm{IQR}=$ interquartile range, with 25 and $75 \%$ values displayed; $\mathrm{TST}=$ tuberculin skin test; $\mathrm{TB}=$ tuberculosis

cAll risk variables were self-reported

${ }^{\mathrm{d}}$ The number of contacts included varies slightly from the numbers presented in the heading for some characteristics because 14 contacts were missing TNF results, 38 contacts were missing IFN results, and 23 contacts were missing IL10 results

${ }^{e} P<0.05$ compared with the referent (first) group within each variable; ${ }^{f} P=0.05-0.20$ compared with the referent (first) group 
Table 4 Median (IQR) ${ }^{\mathrm{b}}$ IFN- $\gamma^{\mathrm{b}}$ Responses to M. tuberculosis Culture Filtrate Protein in pg/ml By Clinical and Epidemiologic Characteristics and Outcome Group

\begin{tabular}{|c|c|c|c|c|c|}
\hline \multirow[t]{2}{*}{ Characteristic $^{c}$} & \multicolumn{3}{|c|}{ Contact Outcome Group } & \multirow{2}{*}{$\begin{array}{l}\text { P-value } \\
\text { Co-prevalent TB } \\
\text { vs. Not TB } \\
\end{array}$} & \multirow{2}{*}{$\begin{array}{l}\text { P-value } \\
\text { Incident TB } \\
\text { vs. Not TB } \\
\end{array}$} \\
\hline & $\begin{array}{l}\text { Co-prevalent TBd } \\
(\underline{n}=41)\end{array}$ & $\begin{array}{l}\text { Incident } \mathrm{TB}^{\mathrm{d}} \\
\underline{(\underline{n}=19)} \\
\end{array}$ & $\begin{array}{l}\text { Not TB } \\
(\underline{n}=1212) \\
\end{array}$ & & \\
\hline All & $231(68-549)$ & $88(0-445)$ & $27(0-349)$ & $<0.001$ & 0.15 \\
\hline \multicolumn{6}{|l|}{ Age (yr) } \\
\hline $0-14$ & $313(101-857)$ & 4994 (4994-4994) & $0(0-294)$ & 0.08 & 0.29 \\
\hline $15-24$ & $336(90-597)$ & $474(153-593)^{f}$ & $32(0-564)^{f}$ & 0.01 & 0.01 \\
\hline $25-44$ & $231(34-410)$ & $0(0-42)$ & $31(0-341)^{e}$ & 0.08 & 0.41 \\
\hline $45-64$ & $125(6-549)$ & $51(0-66)$ & $23(0-309)^{f}$ & 0.12 & 0.65 \\
\hline$>=65$ & $N A^{a}$ & $N A^{a}$ & $77(0-275)^{f}$ & $N A^{a}$ & $N A^{a}$ \\
\hline \multicolumn{6}{|l|}{ Sex } \\
\hline Male & $410(101-562)$ & $109(0-593)$ & $28(0-372)$ & 0.001 & 0.07 \\
\hline Female & $231(69-336)$ & $0(0-154)$ & $27(0-320)$ & 0.049 & 0.57 \\
\hline \multicolumn{6}{|l|}{ Race/Ethnicity } \\
\hline Black & $175(45-644)$ & $50(0-154)$ & $14(0-262)$ & 0.15 & 0.31 \\
\hline Non-black & $336(69-549)$ & $356(0-593)^{f}$ & $41(0-484)^{\mathrm{e}}$ & $<0.001$ & 0.31 \\
\hline \multicolumn{6}{|l|}{ Birthplace } \\
\hline US/Canada & $90(0-430)$ & $46(0-171)$ & $15(0-282)$ & 0.79 & 0.56 \\
\hline Other & $373(178-573)^{e}$ & $474(109-593)^{f}$ & $65(0-692)^{e}$ & $<0.001$ & 0.10 \\
\hline BCG+ & $125(125-3105)$ & $445(0-593)$ & $51(0-594)$ & 0.01 & 0.25 \\
\hline BCG- & $303(6-549)$ & $50(0-154)^{f}$ & $17(0-285)^{e}$ & 0.03 & 0.36 \\
\hline \multicolumn{6}{|l|}{ HIV status ${ }^{\mathrm{b}}$} \\
\hline HIV negative & $336(101-549)$ & $80(0-401)$ & $15(0-309)$ & $<0.001$ & 0.31 \\
\hline HIV unknown & $132(0-644)$ & $2530(66-4994)$ & $32(0-372)$ & 0.47 & 0.16 \\
\hline \multicolumn{6}{|l|}{ Diabetes mellitus } \\
\hline Yes & $N A^{a}$ & $0(0-445)$ & $49(0-259)$ & 0.5584 & 0.56 \\
\hline No & $283(90-549)$ & $109(0-593)$ & $24(0-349)$ & $<0.001$ & 0.07 \\
\hline \multicolumn{6}{|l|}{ Steroids $^{b}$} \\
\hline Yes & $34(0-69)$ & NA & $1(0-252)$ & 1.000 & NA \\
\hline No & $336(113-556)^{f}$ & $88(0-445)$ & $25(0-347)$ & $<0.001$ & 0.15 \\
\hline \multicolumn{6}{|c|}{ Smoking past 6 months } \\
\hline Yes & $358(6-598)$ & $127(0-593)$ & $29(0-316)$ & 0.56 & 0.10 \\
\hline No & $231(101-410)$ & $55(0-131)$ & $23(0-362)$ & $<0.001$ & 1.0 \\
\hline \multicolumn{6}{|l|}{ Heavy alcohol ${ }^{b}$} \\
\hline Yes & $0(0-562)$ & $33(0-211)$ & $35(0-368)$ & 0.57 & 1.0 \\
\hline No & $336(101-549)$ & $132(0-593)$ & $23(0-341)$ & $<0.001$ & 0.11 \\
\hline \multicolumn{6}{|l|}{ IV drugs ${ }^{b}$} \\
\hline Yes & $68(68-68)$ & $0(0-0)$ & $15(0-181)$ & 0.32 & 0.32 \\
\hline No & $336(101-549)$ & $109(0-445)$ & $26(0-348)$ & $<0.001$ & 0.09 \\
\hline \multicolumn{6}{|l|}{ Street drugs ${ }^{b}$} \\
\hline Yes & $35(68-562)$ & $0(0-51)$ & $30(0-290)$ & 0.15 & 0.56 \\
\hline No & $283(95-549)$ & $154(0-593)^{f}$ & $24(0-362)$ & $<0.001$ & 0.07 \\
\hline
\end{tabular}

${ }^{\mathrm{a}} \mathrm{NA}$ (not applicable) in place of a cytokine response designates that there were no specimens for testing; NA in place of a $p$-value designates that a comparison could not be made

${ }^{\mathrm{b}}$ Co-prevalent TB defined as TB diagnosis before or $<30$ days after blood draw; Incident TB defined as TB diagnosis $>30$ days after blood draw; IFN- $\gamma=$ Interferon gamma; Steroids = current steroid use; IV drug = current intravenous drug use; HIV=Human Immunodeficiency Virus; Heavy alcohol = current consumption of $\geq 12$ beers or $>1$ bottle of wine or $>1$ pint of hard liquor per week; Street drugs = current use of drugs other than intravenous acquired without a prescription; IQR $=$ interquartile range, with 25 and $75 \%$ values displayed; TST = tuberculin skin test; TB = tuberculosis

${ }^{c}$ All risk variables were self-reported

${ }^{d}$ The number of contacts included varies slightly from the numbers presented in the heading for some characteristics because 14 contacts were missing TNF results, 38 contacts were missing IFN results, and 23 contacts were missing IL10 results

e $P<0.05$ compared with the referent (first) group within each variable; ${ }^{f} P=0.05-0.20$ compared with the referent (first) group 
Table 5 Multivariate Analysis of Risk factors for Tuberculosis Among Contacts

\begin{tabular}{|c|c|c|c|c|}
\hline \multirow[t]{2}{*}{ Risk Factor $^{\mathrm{b}}$} & \multicolumn{2}{|l|}{ Co-prevalent TB ${ }^{\mathrm{a}}$} & \multicolumn{2}{|l|}{ Incident TBa } \\
\hline & $\begin{array}{l}\text { Odds ratio } \\
(95 \% \mathrm{Cl}) \\
\end{array}$ & $\underline{\text { P-value }}$ & $\begin{array}{l}\text { Odds ratio } \\
(95 \% \mathrm{Cl}) \\
\end{array}$ & $\underline{P \text {-value }}$ \\
\hline \multicolumn{5}{|l|}{ Age group (yrs) } \\
\hline $15-24$ vs. $0-14$ & $0.22(0.07-0.70)$ & 0.01 & & \\
\hline $25-44$ vs. $0-14$ & $0.12(0.04-0.39)$ & $<0.001$ & & \\
\hline $45-64$ vs. $0-14$ & $0.27(0.09-0.83)$ & 0.02 & & \\
\hline \multicolumn{5}{|l|}{$>=65$ vs. $0-14$} \\
\hline \multicolumn{5}{|l|}{ Gender } \\
\hline Female vs. Male & & & $0.24(0.07-0.85)$ & 0.03 \\
\hline \multicolumn{5}{|l|}{ Race/ethnicity } \\
\hline \multicolumn{5}{|l|}{ Non-Black vs. Black } \\
\hline \multicolumn{5}{|l|}{ Birthplace } \\
\hline Foreign vs. US/Canada-born & $0.33(0.15-0.72)$ & 0.005 & & \\
\hline \multicolumn{5}{|l|}{ Diabetes } \\
\hline \multicolumn{5}{|l|}{ No vs. yes } \\
\hline \multicolumn{5}{|l|}{ Steroid use $e^{a}$} \\
\hline \multicolumn{5}{|l|}{ No vs. yes } \\
\hline \multicolumn{5}{|l|}{ Smoking past 6 months } \\
\hline No vs. yes & & & $0.19(0.07-0.56)$ & 0.003 \\
\hline \multicolumn{5}{|l|}{ Heavy alcohol ${ }^{a}$} \\
\hline \multicolumn{5}{|l|}{ No vs. Yes } \\
\hline \multicolumn{5}{|l|}{ IV drugs $^{a}$} \\
\hline \multicolumn{5}{|l|}{ No vs. yes } \\
\hline \multicolumn{5}{|l|}{ Street drugs ${ }^{a}$} \\
\hline \multicolumn{5}{|l|}{ No vs. yes } \\
\hline Hours of exposure & $1.001(1.000-1.001)$ & & & 0.008 \\
\hline \multicolumn{5}{|l|}{ IFN- $\gamma^{a}$ or TNF- $a^{a}$ responses or both } \\
\hline$>$ median vs. neither $>$ median & $5.0(1.49-16.9)$ & 0.010 & & \\
\hline
\end{tabular}

${ }^{a}$ Co-prevalent TB defined as TB diagnosis before or $<30$ days after blood draw; Incident TB defined as TB diagnosis $>30$ days after blood draw; IFN- $\gamma=$ Interferon gamma; TNF-a = Tumor necrosis factor alpha; Steroids = current steroid use; IV drug = current use of intravenous drugs; HIV=Human Immunodeficiency Virus; Heavy alcohol $=$ current consumption of $>12$ beers or $>1$ bottle of wine or $>1$ pint of hard liquor per week; Street drugs = current use of drugs other than intravenous acquired without a prescription; TST tuberculin skin test, TB tubērculosis

${ }^{\mathrm{b}}$ All risk variables were self-reported

with PPV for all exposed contacts (1.5\%), contacts with a positive TST (3\%), or young age alone (1\%). Of note, these algorithms are based on very small samples, and there was wide variability around the point estimates. Further studies with a larger sample size are needed to more fully explore the PPV for incident TB diagnosis of algorithms which combine epidemiologic and immunologic factors.

The PPV for TB disease of CFPS TNF- $\alpha$, IFN- $\gamma$, and IL10 responses singly and in combination without considering epidemiologic factors was considerably lower (4-6\%); however, this is in fact double or triple the expected disease rate $(1-3 \%)$ among exposed contacts [20, 28-30]. Further, CFPS TNF- $\alpha$, IFN- $\gamma$, and IL10 responses singly and in combination had high NPVs for both co-prevalent TB (97-99\%) and incident TB (99$100 \%$ ), indicating a potential for using these responses as a tool to identify a subgroup of contacts with a high certainty of not currently having TB nor of being diagnosed with $\mathrm{TB}$ in the future.

Our findings provide evidence that TNF- $\alpha$ and IFN- $\gamma$ have the potential to help predict the diagnosis of coprevalent TB at the time of contact investigation. TNF- $\alpha$ and IFN- $\gamma$ may thus be useful adjuncts to current diagnostic methods, which consist primarily of symptombased screening with chest radiograph followed by sputum examination and culture, and can take from days to weeks to complete. On the basis of our findings, elevation of either TNF- $\alpha$ or IFN- $\gamma$ responses should heighten suspicion of TB, particularly if both are elevated, the 
Table 6 Algorithms for predicting TB among contacts based on cytokine CF responses and clinical symptoms. A. Co-prevalent TB ${ }^{a}$ versus Not TB ${ }^{\mathrm{a}}$

\begin{tabular}{lcc}
\hline Characteristics & Sensitivity (\%) & S \\
\hline ALL $(\mathbf{4 1}$ co-prevalent TB, 1212 not TB) & a \\
TNF > Median & 71 & 49 \\
INF > Median & 79 & 5 \\
IL10 > Median & 59 & 50 \\
All > Median & 37 & 22 \\
Any > Median & 90 & 4 \\
TST- & 0 & 59 \\
Initial TST+ & 88 & 92 \\
Converter & 2 & 5 \\
TST+/Converter & 100 & 89 \\
Age $<15$ years & 17 & 1 \\
Age $>=15$ years & 83 &
\end{tabular}

Specificity (\%) $\mathrm{PPV}^{\mathrm{b}}(\%)(\mathrm{Cl})^{\mathrm{b}}$

$\mathrm{NPV}^{\mathrm{b}}(\%)$

TST+/Converter (41 co-prevalent TB, 595 not TB) ${ }^{\mathrm{a}}$

$\begin{array}{lll}\text { TNF }>\text { Median } & 51 & 51 \\ \text { INF }>\text { Median } & 69 & 50 \\ \text { IL } 10>\text { Median } & 59 & 51 \\ \text { All }>\text { Median } & 29 & 80 \\ \text { Any }>\text { Median } & 83 & 22\end{array}$

TST+/Converter and age $<15$ years $\left(7\right.$ co-prevalent TB, 33 not TB) ${ }^{\mathrm{a}}$

$\begin{array}{lll}\text { TNF }>\text { Median } & 43 & 52 \\ \text { INF }>\text { Median } & 50 & 53 \\ \text { IL10 > Median } & 43 & 53 \\ \text { All }>\text { Median } & 29 & 91 \\ \text { Any }>\text { Median } & 57 & 18\end{array}$

TST $+/$ Converter and age $>=15$ years ( 34 co-prevalent TB, 562 not TB $)^{\mathrm{a}}$

$\begin{array}{lll}\text { TNF }>\text { Median } & 53 & 51 \\ \text { INF }>\text { Median } & 70 & 50 \\ \text { IL10 }>\text { Median } & 59 & 51 \\ \text { All }>\text { Median } & 26 & 79 \\ \text { Any }>\text { Median } & 85 & 22\end{array}$

Contacts with Cough (23 co-prevalent TB, 148 not TB) ${ }^{a}$

$\begin{array}{lll}\text { TNF }>\text { Median } & 83 & 53 \\ \text { INF }>\text { Median } & 86 & 51 \\ \text { IL10 > Median } & 52 & 50 \\ \text { All }>\text { Median } & 30 & 81 \\ \text { Any }>\text { Median } & 100 & 22\end{array}$

Contacts with Fever or Weight Loss (21 co-prevalent TB, 68 not TB) ${ }^{a}$

$\begin{array}{lll}\text { TNF }>\text { Median } & 81 & 53 \\ \text { INF }>\text { Median } & 100 & 52 \\ \text { IL10 > Median } & 65 & 51 \\ \text { All }>\text { Median } & 43 & 85 \\ \text { Any }>\text { Median } & 100 & 19\end{array}$

$\begin{array}{ll}5(3,7) & 98 \\ 5(4,7) & 99 \\ 4(2,6) & 97 \\ 6(3,9) & 97 \\ 4(3,5) & 98 \\ 0 & 100 \\ 7(5,9) & 99 \\ 1(0,7) & 96 \\ 6(5,9) & 100 \\ 5(2,11) & 97 \\ 3(2,4) & \end{array}$

$7(4,10)$

$9(6,12)$

$7(6,11)$

$9(5,15)$

$7(5,10)$

$16(4,40)$

$17(4,42)$

$17(4,42)$

$40(7,83) \quad 86$

$13(4,31) \quad 67$

$\begin{array}{ll}6(4,10) & 95 \\ 8(5,12) & 96 \\ 6(4,10) & 96 \\ 7(3,13) & 95 \\ 6(4,9) & 96\end{array}$

$22(14,32)$

$21(13,31) \quad 96$

$13(7,23) \quad 88$

$20(9,37)$

$17(11,24) \quad 100$

$35(23,51) \quad 90$

$38(26,53) \quad 100$

$28(16,44) \quad 83$

$47(25,71) \quad 83$

$28(18,39) \quad 100$ 
Table 6 Algorithms for predicting TB among contacts based on cytokine CF responses and clinical symptoms. A. Co-prevalent TB ${ }^{a}$ versus Not TBa (Continued)

\begin{tabular}{|c|c|c|c|c|}
\hline Characteristics & Sensitivity (\%) & Specificity (\%) & $\mathrm{PPV}^{\mathrm{b}}(\%)(\mathrm{Cl})^{\mathrm{b}}$ & NPV $(\%)$ \\
\hline \multicolumn{5}{|c|}{ Contacts with Immune Compromising Conditions (34 co-prevalent TB, 844 not TB) ${ }^{a}$} \\
\hline TNF $>$ Median & 76 & 51 & $6(4,9)$ & 98 \\
\hline INF $>$ Median & 85 & 51 & $7(4,9)$ & 99 \\
\hline IL10 > Median & 62 & 51 & $5(3,7)$ & 97 \\
\hline All > Median & 44 & 80 & $8(5,13)$ & 97 \\
\hline Any > Median & 94 & 121 & $5(3,6)$ & 99 \\
\hline
\end{tabular}

${ }^{a}$ The number of contacts included varies slightly from the numbers presented in the subheading for some characteristics because 14 contacts were missing TNF results (all 14 not TB), 38 contacts were missing IFN results ( 2 co-prevalent TB and 36 not TB), and 23 contacts were missing IL10 results ( 2 co-prevalent TB and 21 not TB)

${ }^{\mathrm{b}}$ Co-prevalent TB defined as TB diagnosis before or $<30$ days after blood draw; TNF- $a$ Tumor necrosis factor alpha, TST tuberculin skin test, TB tuberculosis, Immune Compromising Conditions = diabetes, kidney failure, cancer, chemotherapy, organ transplant, or current steroid use; CF culture filtrate, PPV positive predictive value, NPV negative predictive value, $\mathrm{Cl}$ confidence interval

patient has clinical symptoms of TB, a positive TST, or is of young age. Further, the absence of TNF- $\alpha$ elevation in a single measurement soon after TB exposure could be used to define a group at low risk for TB.

The role of TNF- $\alpha$ as a pro-inflammatory cytokine essential to host defense against TB is well-established [3-5, 31, 32]. Previous studies have evaluated cytokine responses among patients with TB at various times after treatment initiation [5, 24-26]. The majority of studies reported that TNF- $\alpha$ levels are higher among TB patients than control subjects evaluated soon after treatment initiation [5, 24-26], with a subsequent decline to levels similar to those of control subjects associated with successful resolution of disease [5]. In one report, the ratio of TNF- $\alpha$ to IL-10 was determined to be useful in distinguishing latent TB infection from active disease [32]. In our study, contacts who were diagnosed with incident TB over a 4year follow-up had substantially higher CFPS TNF- $\alpha$ responses at baseline than contacts who remained disease free. This novel finding suggests that elevation in CFPS TNF- $\alpha$ responses could help predict subsequent development of active TB. This univariate finding was not supported by the multivariate analysis, however, and thus is not conclusive. The multivariate result may have been influenced by the very small number of persons in our study who subsequently developed TB. Additional studies with larger numbers are needed to further explore the possible role of elevation in CFPS TNF- $\alpha$ responses as a predictor for subsequent development of active TB. Our findings provide new information revealing that measurement of TNF- $\alpha$ might be helpful in predicting which exposed contacts will be diagnosed with $\mathrm{TB}$ at an earlier time than current diagnostic algorithms [33]. Further, these findings put into new context the increase in TNF- $\alpha$ among persons with previously treated TB at the time of relapse observed in an earlier report [5] by raising the question of whether measurement of TNF- $\alpha$ could help to predict TB relapse.
IFN- $\gamma$ is known to play an important role in host defense against $M$. tuberculosis. In the majority of studies, IFN- $\gamma$ levels have been reported to be low soon after TB diagnosis, with a subsequent increase reflecting successful resolution of disease [3, 25, 34]. IFN- $\gamma$ assays lack optimal sensitivity for TB disease, however, with elevations among only an estimated $70-90 \%$ of active TB cases $[9,26]$. Furthermore, although measurement of IFN- $\gamma$ responses can be useful for diagnosing both latent TB infection and TB disease, current assays cannot discriminate between the 2 conditions. In our study, IFN- $\gamma$ responses were significantly higher among contacts with co-prevalent $\mathrm{TB}$ compared with contacts without $\mathrm{TB}$, but were similar for contacts with co-prevalent $\mathrm{TB}$ and those who were TST-positive without TB. Our findings indicate that measuring IFN- $\gamma$ responses might be helpful in predicting active TB weeks to months earlier than current diagnostic algorithms (which rely on follow-up skin testing of contacts who were initially skin test negative 8-10 weeks after exposure) [34] and in identifying which exposed contacts have been infected with $M$. tuberculosis, but cannot identify which contacts with latent TB infection will subsequently develop TB.

A tool that enhances identification of persons at highest risk for TB disease might enable health departments to better prioritize public health investigations of persons exposed to infectious TB. Thus, interventions could be targeted at the limited number of persons truly at risk, thereby increasing the efficiency and effectiveness of public health prevention measures and reducing costs. Our findings indicate a potential role for both CFPS TNF- $\alpha$ and IFN $-\gamma$ responses as adjunctive diagnostic tools for TB disease. IFN- $\gamma$ response assays are already in clinical use, but primarily for diagnosing latent TB infection. Although our findings are intriguing, further evidence for the clinical utility of incorporating CFPS TNF- $\alpha$ responses into diagnostic algorithms for TB as well as development of a TB-specific commercial assay for TNF- $\alpha$ will be necessary 
Table 7 Algorithms for predicting TB among contacts based on cytokine CF responses and clinical symptoms. B. Incident TB ${ }^{a}$ versus Not $\mathrm{TB}^{\mathrm{a}}$

\begin{tabular}{|c|c|}
\hline Characteristics & Sensitivity (\%) \\
\hline \multicolumn{2}{|c|}{ ALL (19 incident TB, 1212 not TB) ${ }^{a}$} \\
\hline TNF > Median & 68 \\
\hline INF > Median & 67 \\
\hline IL10 > Median & 61 \\
\hline All > Median & 47 \\
\hline Any > Median & 79 \\
\hline TST- & 0 \\
\hline Initial TST+ & 79 \\
\hline Converter & 27 \\
\hline TST+/Converter & 100 \\
\hline Age $<15$ years & 5 \\
\hline Age $>=15$ years & 95 \\
\hline \multicolumn{2}{|c|}{ TST+/Converter (19 incident TB, 595 not TB) ${ }^{a}$} \\
\hline TNF > Median & 68 \\
\hline INF > Median & 50 \\
\hline IL10 > Median & 61 \\
\hline All > Median & 37 \\
\hline Any $>$ Median & 74 \\
\hline
\end{tabular}

TST+/Converter and age $<15$ years $\left(1\right.$ incident TB, 33 not TB) ${ }^{\mathrm{a}}$
TNF $>$ Median 100

INF $>$ Median 100

IL10 $>$ Median $\quad 100$

All > Median

100

100

Any > Median
Specificity (\%)

49

51

50

80

22

49

59

92

51

89

11

\section{1}

50

51

80

22

TST+/Converter and age $>=15$ years $(18 \text { incident TB, } 562 \text { not TB })^{\mathrm{a}}$

TNF > Median

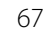

INF $>$ Median

47

IL10 > Median

59

All > Median

Any $>$ Median

Contacts with Cough (5 incident TB, 148 not TB) ${ }^{a}$
TNF $>$ Median

INF > Median

IL10 > Median

All > Median

Any $>$ Median
80

60

60

40

80

Contacts with Fever or Weight Loss (1 incident TB, 68 not TB)

TNF > Median

INF > Median

IL10 > Median

All > Median

Any $>$ Median
100

100

100

100

100
$\mathrm{PPV}^{\mathrm{b}}(\%)$

$(95 \% \mathrm{Cl})^{\mathrm{b}}$

$2(1,4)$

$2(1,4)$

$2(1,3)$

$4(2,7)$

$2(1,3)$

0

$3(2,5)$

$4(1,11)$

$3(2,5)$

$1(0,5)$

$2(1,3)$

$\mathrm{NPV}^{\mathrm{b}}(\%)$

$4(0,20)$

$\begin{array}{ll}4(2,7) & 98 \\ 3(1,6) & 97 \\ 4(2,7) & 98 \\ 5(2,11) & 97 \\ 3(2,5) & 96\end{array}$

$5(2,14) \quad 99$

$4(1,12) \quad 97$

$4(2,7) \quad 97$

$7(1,24) \quad 98$

$3(1,9) \quad 97$

$\begin{array}{ll}3(0,18) & 100 \\ 3(0,18) & 100 \\ 3(0,17) & 100 \\ 9(0,43) & 100 \\ 2(0,11) & 100\end{array}$


Table 7 Algorithms for predicting TB among contacts based on cytokine CF responses and clinical symptoms. B. Incident TB ${ }^{a}$ versus Not TB ${ }^{a}$ (Continued)

\begin{tabular}{|c|c|c|c|c|}
\hline Characteristics & Sensitivity (\%) & Specificity (\%) & $\begin{array}{l}\text { PPV }^{\mathrm{b}}(\%) \\
\left(95 \%()^{b}\right.\end{array}$ & NPV (\%) \\
\hline \multicolumn{5}{|c|}{ 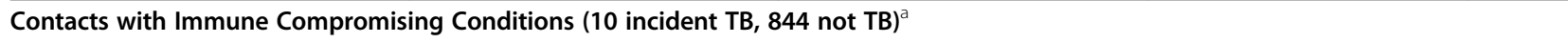 } \\
\hline TNF > Median & 80 & 51 & $2(1,4)$ & 99 \\
\hline INF $>$ Median & 89 & 51 & $2(1,4)$ & 100 \\
\hline IL10 > Median & 89 & 51 & $2(1,4)$ & 100 \\
\hline All > Median & 70 & 80 & $4(2,8)$ & 100 \\
\hline Any $>$ Median & 90 & 21 & $1(1,3)$ & 99 \\
\hline
\end{tabular}

before measurement of CFPS TNF- $\alpha$ responses can be routinely incorporated into clinical care.

Our study provides new evidence to support immunemediated mechanisms for the link between immune compromising medical conditions such as diabetes and steroid use, excess alcohol use, smoking, and illicit drug use and increased risk for TB. Persons with immunocompromising medical conditions are known to be at higher risk for TB than normal hosts [35]. Excess alcohol use, cigarette smoking, illicit drug use, and older or younger age have also been linked to increased TB risk, with immune-mediated mechanisms postulated [36-44]. Excess alcohol use is associated with decreased production of TNF- $\alpha[37,38]$, and exposure to cigarette smoke has been associated in mouse models with decreased production of TNF- $\alpha$ and IFN- $\gamma$ as well as increased production of IL-10 [39, 40]. In our study, CFPS TNF- $\alpha$ and IFN- $\gamma$ responses were lower for persons with immunocompromising medical conditions and substance use than for those without such conditions, indicating that these conditions might affect the human immune response through both TNF- $\alpha$ and IFN- $\gamma$ cytokine response pathways. Furthermore, whereas CFPS TNF- $\alpha$ and IFN- $\gamma$ responses were higher among contacts with TB compared with contacts without TB for normal hosts, no difference in responses was observed for either cytokine among contacts with immunocompromising medical conditions or substance abuse, indicating that these conditions might blunt both the TNF- $\alpha$ and IFN- $\gamma$ immune responses to TB.

CFPS TNF- $\alpha$ responses among our study population were substantially higher among persons of black versus non-black race across all outcome groups. These novel findings are intriguing, particularly in light of established differences in TB rates among persons in the United States by race/ethnicity [45]. Further studies are needed to validate these findings, and to determine whether variation in CFPS TNF- $\alpha$ cytokine responses by race is associated with functional changes which affect susceptibility, immune response, and TB risk following M. tuberculosis exposure. These findings also have implications for considering race/ethnicity in vaccine trials using cytokine-based surrogate markers.

Our study has certain limitations. Blood specimens were not obtained at the same time point after exposure for all contacts, which might affect variability of responses; not all eligible contacts agreed to participate in the immunologic study; the number of contacts with $\mathrm{TB}$ was relatively limited; and we did not account for clustering of TB by index case in the multivariable analysis. Although we cannot exclude the possibility that our study failed to detect one or more cases among contacts no longer under follow-up, the number missed is expected to be quite small since follow-up completeness was $100 \%$ at 4 years, and $94 \%$ at 5 years; further, $92 \%$ of all cases occurred before or within the first year after the end of exposure, followed by a steep decline in subsequent years [20]. Therefore, our epidemiological data suggest that very few secondary cases among contacts were missed either before or after the 5-year post exposure time point. Our findings that most secondary cases occur soon after exposure and could not have been prevented even with timely contact investigation [20], the fact that only one third of contacts with LTBI completed treatment [46], and evidence that most contacts who initiated treatment did so $>3$ months after exposure $(\mathrm{M}$ Reichler personal communication), when the risk of progression to tuberculosis is already lower, suggest that the impact of LTBI treatment on the rates and timing of tuberculosis in our study is not expected to be large. Despite the limitations, substantial epidemiologic data of good quality were collected in addition to CFPS cytokine responses, a strength of the study.

\section{Conclusions}

Our study findings confirm that IFN- $\gamma$ and TNF- $\alpha$ are immune correlates of TB disease, and demonstrate that 
cytokine concentrations and epidemiologic factors at the time of contact investigation may predict co-prevalent tuberculosis, and may also be useful to rule out development of active TB based on absence of cytokine elevation In our study, we observed differences in CFPS cytokine responses by age, race, underlying medical condition, and substance abuse, highlighting the value of examining immunologic correlates of $M$. tuberculosis infection and disease in the context of clinical and epidemiologic factors. Further studies are needed to validate these findings and to explore the role of other cytokines and chemokines involved in immune defense against $M$. tuberculosis.

\begin{abstract}
Abbreviations
TB: Tuberculosis; TST: Tuberculin skin test; TST: Tuberculin skin test-positive; TST-: Tuberculin skin test-negative; IV: Intravenous; HIV: Human Immunodeficiency Virus; IQR: Interquartile range; Cl: Confidence interval; PHA: Phytohemagglutinin; TNF-a: Tumor necrosis factor alpha; IFNY: Interferon gamma; IL-10: Interleukin 10; M. tb: Mycobacterium tuberculosis; CF: Culture filtrate; CFP: Culture filtrate protein; CFPS: Culture filtrate proteinstimulated; US: United States; PPV: Positive predictive value; NPV: Negative predictive value
\end{abstract}

\section{Acknowledgements \\ Task Order Two Team. \\ The Tuberculosis Epidemiological Studies Consortium (TBESC) Task Order 2 study sites, investigators, and study coordinators are as follows: Arkansas Department of Health, Little Rock, Arkansas (I. Bakhtawar, C. LeDoux); Respiratory Health Association of Metropolitan Chicago and Rush University (J. McAuley, J. Beison); University of British Columbia (M. Fitzgerald, M. Naus, M. Nakajima); Columbia University (N. Schluger, Y. Hirsch-Moverman, J. Moran); Emory University (H. Blumberg, J. Tapia, L. Singha); University of Manitoba (E. Hershfeld, B. Roche); New Jersey Medical School National Tuber- culosis Center (B. Mangura, A. Sevilla); Vanderbilt University and Tennessee Department of Health (T. Sterling, T. Chavez-Lindell, F. Maruri); Maryland De- partment of Health, Baltimore, Maryland (S. Dorman, W. Cronin, E. Munk). The Centers for Disease Control and Prevention Task Order 2 data management team is as follows: A. Khan, Y. Yuan, B. Chen, F. Yan, Y. Shen, H. Zhao, H. Zhang, P. Bessler, M. Fagley, M. Reichler. \\ The Task Order 2 Protocol Team is as follows: M. Reichler (Chair), T. Sterling (Co-chair), J. Tapia, C. Hirsch, and C. Luo. \\ Other Acknowledgements. We thank Thomas Navin, Andrew Vernon, and Deron Burton for helpful guidance and input into scientific and administrative aspects of the project.}

\section{Disclaimer}

The findings and conclusions in this report are those of the authors and do not necessarily represent the official position of the Centers for Disease Control and Prevention.

\section{Authors' contributions}

$\mathrm{CH}$ performed all cytokine laboratory testing. $\mathrm{YY}, \mathrm{AK}$, and $\mathrm{MR}$ analyzed the data. MR, TS, SD, NS, CH, AK, and YY interpreted the data. MR and TS were major contributors in writing the manuscript. MR, TS, SD, NS, CH, AK, and $Y Y$ read and approved the final manuscript.

\section{Funding}

This work was supported by the US Centers for Disease Control and Prevention (CDC) Tuberculosis Epidemiologic Studies Consortium, a federal public health research collaboration between investigators at academic institutions, health departments, and CDC [22]. All collaborators jointly designed the study, developed the protocol, implemented the study, analyzed and interpreted the data, and prepared the manuscript. The findings and conclusions in this report are those of the authors and do not necessarily represent the official position of the Centers for Disease Control and Prevention.

\section{Availability of data and materials}

The datasets generated and analyzed during the current study are not publicly available due to Centers for Disease Control and Prevention Institutional Review Board restrictions, but copies of all data output used to generate this report are available from the corresponding author upon reasonable request.

\section{Ethics approval and consent to participate}

The protocol was approved by the institutional review boards at the Centers for Disease Control and Prevention (FWA00001413), Case Western Reserve University (FWA00004428), Columbia University (FWA00002636), Emory University (FWA0005792), Vanderbilt University (FWA00005756), New Jersey Department of Health (FWA00004020), Rush University (FWA00000482), University of Arkansas (FWA00002205), and Maryland Department of Health (FWA00002813), and by the research ethics boards of the University of British Columbia (FWA00000668) and the University of Manitoba (FWA00002049).

Participants were enrolled using written informed consent.

\section{Consent for publication}

Not applicable.

\section{Competing interests}

The authors declare that they have no competing interests.

\section{Author details}

${ }^{1}$ National Center for HIV/AIDS, Viral Hepatitis, STD, and TB Prevention, Division of Tuberculosis Elimination, Centers for Disease Control and Prevention, Mailstop E-10, 1600 Clifton Road NE, 30329-4027 Atlanta, GA, USA. ${ }^{2}$ Department of Medicine, Case Western Reserve University, Cleveland, $\mathrm{OH}$, USA. ${ }^{3}$ Department of Medicine, Johns Hopkins University, Baltimore, MD, USA. ${ }^{4}$ Department of Medicine, Columbia University, New York, NY, USA.

${ }^{5}$ Department of Medicine, Vanderbilt University, Nashville, TN, USA.

Received: 26 August 2019 Accepted: 22 June 2020

Published online: 31 July 2020

\section{References}

1. World Health Organization. WHO Global Tuberculosis Report 2019. Geneva: World Health Organization; 2019

2. Sia JK, Rengarajan J. Immunology of mycobacterium tuberculosis infection. Microbiol Spectrum. 2019;7(4). https://doi.org/10.1128/microbiolspec.GPP30022-2018.

3. Hirsch CS, Toosi Z, Othieno C, et al. Depressed T-cell interferon gamma responses in pulmonary tuberculosis: analysis of underlying mechanisms and modulation with therapy. J Infect Dis. 1999;180(6):2069-73.

4. Ellner JJ. Regulation of the human immune response during tuberculosis. J Lab Clin Med. 1997;130(5):469-75.

5. Ellner JJ, Hirsch CS, Whalen C. Correlates of protective immunity to Mycobacterium tuberculosis infection in humans. Clin Infect Dis. 2000; 30(Supplement 3):S279-82.

6. Moreira-Teixeria $\mathrm{L}$, et al. T-cell derived IL-10 impairs host resistance to Mycobacterium tuberculosis infection. J Immunol. 2017;199:613-23.

7. Othieno C, Hirsch CS, Hamilton BD, Wilkinson K, Ellner JJ, Toosi Z. Interaction of mycobacterium tuberculosis-induced transforming growth factor beta 1 and interleukin 10. Infect Immun. 1999:67(11):5730-5.

8. Whalen CC, Chiunda A, Zalwango $S$, et al. Immune correlates of acute mycobacterium tuberculosis infection in household contacts in Kampala, Uganda. Am J Trop med Hyg. 2006;75(1):55-61.

9. Pai M, Riley LW, Colford JM Jr. Interferon-gamma assays in the immunodiagnosis of tuberculosis: a systematic review. Lancet Infect Dis. 2004:4:761-76.

10. Mazurek G, Jereb J, Vernon A, et al. Updated guidelines for using interferon gamma release assays to detect Mycobacterium tuberculosis infection United States, 2010 (Mazurek G, Jereb J, Vernon A, et al). MMWR Recommend Rep. 2010;59(RR-05):1-25.

11. Arend SM, Thijsen SFT, Leyten EMS, et al. Comparison of two interferonassays and tuberculin skin test for tracing tuberculosis contacts. Am J Respir Crit Care Med. 2007;175:618-27.

12. Diehl R, Goletti $D$, Ferrara $G$, et al. Interferon gamma release assays for the diagnosis of latent mycobacterium tuberculosis infection: a systematic review and meta-analysis. Eur Respir J. 2011;37(1):88-99. 
13. Pai M, Kunimoto $D$, Jamieson $F$, Menzies D. Diagnosis of latent tuberculosis infection. Canadian TB Standards, 7th Edition. Can Respir J. 2013;20(Suppl A): 23A.

14. Corral H, Paris SC, Marin ND, et al. IFN-gamma response to mycobacterium tuberculosis, risk of infection and disease in household contacts of tuberculosis patients in Colombia. PLoS One. 2009:4(12):e8257.

15. Diel R, Loddenkemper R, Meywald-Walter K, Niemann S, Nienhaus A. Predictive value of a whole blood IFN-gamma assay for the development of active tuberculosis disease after recent infection with mycobacterium tuberculosis. Am J Respir Crit Care Med. 2008;177(10):1164-70.

16. Machingaidze $\mathrm{S}$, Verver $\mathrm{S}$, Mulenga $\mathrm{H}$, et al. Predictive value of recent quantiFERON conversion for tuberculosis disease in adolescents. Am J Respir Crit Care Med. 2012;186:1051-6.

17. Mahomed H, Hakridge T, Verver S, et al. The tuberculin skin test versus QuantiFERON TB gold in predicting tuberculosis disease in an adolescent cohort study in South Africa. PLoS One. 2011;6(3):e17984.

18. Diehl R, Loddenkemper $R$, Nienhaus A. Predictive value of interferon-gamma release assays and tuberculosis skin testing for progression from latent TB infection to disease state: a meta-analysis. Chest. 2012:142(1):63-75.

19. Zak DE, Penn-Nicholson A, Scriba TJ, et al. A prospective blood RNA signature for tuberculosis disease risk. Lancet. 2016;387(10035):2312-22.

20. Reichler MR, Khan A, Sterling TR, et al. Risk and timing of tuberculosis among close contacts of persons with infectious tuberculosis. J Infect Dis. 2018;218:1000-8.

21. Reichler MR, Khan A, Sterling TR, et al. Risk factors for tuberculosis and effect of preventive therapy among close contacts of persons with infectious tuberculosis. Clin Infect Dis. 2019. https://doi.org/10.1093/cid/ciz438.

22. Katz D, Albalak R, Wing JS, Combs V, Consortium TES. Setting the agenda: a new model for collaborative tuberculosis epidemiologic research. Tuberculosis. 2007:87:1-6.

23. Hirsch-Moverman Y, Cronin W, Chen B, Moran JA, Munk E, Reichler MR. HIV counseling and testing in tuberculosis contact investigations in the United States and Canada. IJTLD. 2015;19(8):943-53.

24. Nemeth J, Winkler $H$, Boeck $L$, et al. Specific cytokine patterns of pulmonary tuberculosis in Central Africa. Clin Immunol. 2011;138:50-9.

25. Hussain R, Kaleem A, Shahid F, et al. Cytokine profiles using whole-blood assays can discriminate between tuberculosis patients and healthy endemic controls in a BCG-vaccinated population. J Immunol Methods. 2002;264:95108.

26. Kellar KL, Gehrke J, Weis SE, et al. Multiple cytokines are released when blood from patients with tuberculosis is stimulated with mycobacterium tuberculosis antigens. PLoS One. 2011;6(11):e26545.

27. Petruccioli E, Scriba TJ, Petrone L, et al. Correlates of tuberculosis risk: predictive biomarkers for progression to active tuberculosis. Eur Respir J. 2016;48(6):1751-63.

28. Reichler MR, Reves R, Bur S, et al. Evaluation of investigations conducted to detect and prevent transmission of tuberculosis. JAMA. 2002;287(8):991-5.

29. Jereb J, Etkind SC, Joglar OT, Moore M, Taylor Z. Tuberculosis contact investigations: outcomes in selected areas of the United States, 1999. Int J Tuberc Lung Dis. 2003;7(Suppl 1):S384-90.

30. Marks S, Taylor Z, Qualls N, et al. Outcomes of contact investigations of infectious tuberculosis patients. Am J Respir Crit Care Med. 2000;162(6): 2033-8

31. Jacobs M, Togbe D, Fremond C, et al. Tumor necrosis factor is critical to control tuberculosis infection. Microbes Infect. 2007:9(5):623-8.

32. Jacobs M, Samarina A, Grivennikov S, et al. Reactivation of tuberculosis by tumor necrosis factor neutralization. Eur Cytokine Netw. 2007:18(1):5-13.

33. Tebruegge M, Dutta B, Donath $\mathrm{S}$, et al. Mycobacteria-specific cytokine responses detect TB infection and distinguish latent from active TB. Am J Crit Care Med. 2015;192(4):485-99.

34. Centers for Disease Control and Prevention. Guidelines for the investigation of contacts of persons with infectious tuberculosis: recommendations from the National Tuberculosis Controllers Association and CDC. MMWR Recommend Rep. 2005;54(RR-15):1-37.

35. Riano F, Arroyo L, Paris S, et al. T cell responses to DosR and Rpf proteins in actively and latently infected individuals from Colombia. Tuberculosis. 2012; 92(2):148-59.

36. Centers for Disease Control and Prevention. Targeted tuberculin testing and treatment of latent tuberculosis infection. MMWR Recommend Rep. 2000; 49(RR-6):1-54.
37. Omidvari K, Casey R, Nelson S, Olariu R, Shellito JE. Alveolar macrophage release of TNF in chronic alcoholics without liver disease. Alcohol Clin Exp Res. 1998;22(3):567-72.

38. Nelson S, Mason C, Bagby G, Summer W. Alcohol, tumor necrosis factor, and tuberculosis. Alcohol Clin Exp Res. 1995;19(1):17-24.

39. Gajalakshmi V, Peto R, Kanaka TS, Jha P. Smoking and mortality from tuberculosis and other diseases in India: retrospective study of 43,000 adult male deaths and 35,000 controls. Lancet. 2003;362:507-15.

40. Davies PD, Yew WW, Ganguly D, et al. Smoking and tuberculosis: the epidemiological association and immunopathogenesis. Trans R Soc Trop Med Hyg. 2006:100:291-8.

41. Shang $S$, Ordway D, Henao-Tamayo $M$, et al. Cigarette smoke increases susceptibility to tuberculosis- evidence from in vivo and in vitro models. Infect Dis. 2011;203(9):1240-8.

42. Feng $Y$, Kong $Y$, Barnes PF, et al. Exposure to cigarette smoke inhibits the pulmonary T-cell response to influenza virus and Mycobacterium tuberculosis. Infect Immun. 2011:79(1):229-37.

43. Lewinsohn DA, Zalwango S, Stein C, et al. Whole blood interferon-gamma responses to Mycobacterium tuberculosis antigens in young household contacts of persons with tuberculosis in Uganda. PLoS One. 2008;3(10): e3407.

44. Kumar NP, Anuradha R, Suresh R, et al. Suppressed type 1, type 2, and type 17 cytokine responses in active tuberculosis in children. Clin Vaccine Immunol. 2011:18(11):1856-64.

45. CDC. Tuberculosis - United States, 2017. MMWR Morb Mortal Wkly Rep. 2018:67(11):317-23.

46. Fiske CT, Yan FX, Hirsch-Moverman Y, Sterling TR, Reichler MR. Risk factors for treatment default in close contacts with latent tuberculosis infection. Int J Tuberc Lung Dis. 2014;18(4):421-7.

\section{Publisher's Note}

Springer Nature remains neutral with regard to jurisdictional claims in published maps and institutional affiliations.
Ready to submit your research? Choose BMC and benefit from:

- fast, convenient online submission

- thorough peer review by experienced researchers in your field

- rapid publication on acceptance

- support for research data, including large and complex data types

- gold Open Access which fosters wider collaboration and increased citations

- maximum visibility for your research: over $100 \mathrm{M}$ website views per year

At BMC, research is always in progress.

Learn more biomedcentral.com/submission 